\title{
MedienPädagogik
}

Zeitschrift für Theorie und Praxis der Medienbildung

\section{Jugend in der globalen Medienkultur}

\author{
Kai-Uwe Hugger
}

Jugend unter Globalisierungsgesichtspunkten zu betrachten, ist für die deutschsprachige Erziehungswissenschaft relativ neu. Zwar wird in den letzten Jahren zunehmend häufiger gefordert, Fragen von Bildung, Sozialisation und Erziehung stärker in der Globalisierungsperspektive zu diskutieren (vgl. etwa Asbrand 2002; Hornstein 2001; Nestvogel 2000; Scheunpflug 2003). Immer noch muss aber festgehalten werden, dass die Erziehungswissenschaft Jugend vor allem als nationale Kategorie versteht. Auf diese Weise geraten auch globale Ausdrucksformen von Jugendkulturen kaum oder gar nicht in den Blick. Die Medien - so will ich auf den folgenden Seiten argumentieren - tragen heute in zentraler Weise dazu bei, dass sich in einer immer mehr globalen Kultur globalisierte Medienszenen von Jugendlichen herausbilden. Diese können wichtige Ausdrucksformen dafür sein, wie die Sinn- und Identitätssuche unter den Bedingungen komplexer Globalisierungsprozesse auf jugendspezifische Weise bewältigt wird. In diesen globalisierten Medienszenen nutzen die Jugendlichen Medien, um globale (Medien-) Kultur in unterschiedlicher Weise und für verschiedene Zwecke in der eigenen Lebenswelt auszuhandeln.

\section{Zum Grundverhältnis von Globalisierung und Medien}

Jenseits einer polaren Diskussion zwischen euphemistischen Globalisierungsbefürwortern und ebenso „radikalen“ Skeptikern, die die Irrelevanz des „G-Wortes“ (für die Pädagogik) behaupten, wird eine versachlichte Globalisierungsdebatte in den Sozialwissenschaften deutlich, die komplex, facettenreich und mit teils stark widersprechenden Argumenten geführt wird. Internationale und transnationale Kräfte der gesellschaftlichen Entwicklung dazu gehören auch die Medien - sind bereits seit längerem Thema der wissenschaftlichen Auseinandersetzung. Eine Unterteilung, die an dieser Stelle nur grob ausfallen kann und die Verästelungen der Diskussion nicht berücksichtigt, kann zwischen klassischen Ansätzen vorgenommen werden und einer „neuen“ Debatte, die seit etwa Anfang der 1990er Jahre zu beobachtenden ist.

Zu den ersteren Ansätzen gehören Theorien der Weltzivilisation. Sie versuchen „regions of civilization“ zu markieren, die durch die Grenzen der modernen Nationalstaaten hindurch gehen oder die schon vor diesen existiert haben. In historischer Sicht wird die Bedeutung des Nationalstaates durch langfristigere 
religiöse, kulturelle und politische Entwicklung relativiert. So entwirft Nelson (1973) das Bild von durchlässigen „civilization complexes“ als generelle kulturelle Muster, die über klar bestimmbare und separierbare politische Gesellschaftsformationen hinaus gehen. Dagegen geht Huntington (1993) eher von der Bestimmbarkeit unterschiedlicher Zivilisationen aus und befürchtet ein „clash of civilizations“. Innerhalb der Zivilisationen differenziert er jedoch kaum. Kritisch ist darüber hinaus anzumerken, dass er sich in seiner Analyse zu wenig den überlappenden Konstellationen oder sozialen Mischverhältnissen widmet. Zu den klassischen Ansätzen können auch Theorien des Weltsystems gezählt werden. Sie richten sich ausdrücklich gegen die Vorstellung von sozialen Räumen als gegeneinander abgrenzbare geographisch-räumliche „Container“. Der einflussreichste Ansatz in diese Richtung wird von Wallerstein (1987) vertreten. Er entwirft das Gegenbild eines „modernen kapitalistischen Weltsystems“, in dem sich alle unterschiedlichen Gesellschaften und ihre Kulturen in einer allumfassenden Arbeitsteilung integrieren müssen. Ausgehend vom Europa des 16. Jahrhunderts setze sich dieses eine Weltsystem in dynamischer Weise immer mehr durch. Kulturelle Differenzen und politische Konjunkturen seien erst im Rahmen der kapitalistischen Weltökonomie erklärbar. Wallerstein vertritt eine monokausale und ökonomistische Argumentationslinie. Globalisierung versteht er vor allem als Institutionalisierung des Weltmarktes. Dieser Ansatz ist in mehrfacher Hinsicht zu kritisieren: So handelt es sich um eine funktionalistischverkürzte Herangehensweise, die jedes Ereignis mit der allumfassenden Struktur des Weltsystems in Verbindung bringt. Darüber hinaus werden in der Analyse kulturelle und politische Faktoren sowie das Handeln von Einzelnen und Gruppen vernachlässigt. Außerdem ist die eher eurozentrische bzw. nordatlantische Perspektive des Weltsystems zu bemängeln. Ein alternativer Blickwinkel, der etwa vom chinesischen oder Hindu-Reich ausgeht, wird nicht in Erwägung gezogen (vgl. Lewis/Wigen 1997).

In der „neuen“ soziologischen Globalisierungsdebatte wird das eher statische und eindimensionale Verständnis von Globalisierung, wie es in den klassischen Ansätzen zum Ausdruck kommt, durch eine dynamische, dialektische und mehrdimensionale Prozess-Vorstellung ersetzt (Featherstone 1990). Als besonders einflussreiche Vertreter gehen Giddens (1990, 1991) und Robertson (1992). Für Giddens ist Globalisierung eine Konsequenz der Moderne. Ihre Globalisierungsdynamik beruht auf drei komplementären Prozessen: 1) time-space distanciation, mit der eine grundlegende Reorganisation von Raum und Zeit, vor allem im Kontext der neuen Informations- und Kommunikationstechnologien, gemeint ist. Dieser Prozess ermöglicht 2) disembedding/reembedding. Damit wird die Herauslösung sozialer Beziehungen aus lokalen Interaktionseinheiten und ihre Restrukturierung über raum-zeitliche Distanzen hinweg angesprochen. Ein Beispiel ist die Frage, wie freundschaftliche Beziehungen zwischen Kontinenten etwa über das Internet gepflegt werden. Die dritte Globalisierungstendenz der Moderne beschreibt Giddens schließlich als (institutional) reflexivity, verstanden als die Anforderung, soziale Praxis ständig hinterfragen und 
auch verändern zu müssen, mit anderen Worten: „das eigene Leben selbst bestimmen zu müssen“ (Beck/Giddens/Lash 1996, S. 322), etwa im Zuge von (unsicheren) Informationen über lebensbedrohliche Risiken wie Aids oder BSE.

Robertson (1992) kritisiert Giddens vor allem deshalb, weil sein Ansatz behauptet, Globalisierung sei eine unmittelbare Folge der Moderne. Dadurch aber - so Robertson - würden wichtige Unterschiede verwischt, so dass die Komplexität des gegenwärtigen Globalisierungsprozesses nicht in vollem Umfang erfasst werden könne. Er plädiert dagegen für ein analytisches Herangehen, das es ermöglicht, Modernität und Globalität in ihrer unabhängigen Bedeutung anzuerkennen. In Abgrenzung zu Giddens’ Analyse entwickelt Robertson sein Modell des „global field“, in dem das Zusammenspiel von vier - mehr oder weniger autonomen - Elementen bzw. Akteuren den Globalisierungsprozess erfasst werden soll: 1) das individuelle Selbst, b) die nationalen Gesellschaften, c) das internationale System von Gesellschaften und d) die Menschheit. Die heutige Welt als ganze, so Robertson (1998, S. 208), habe sich „aus wechselnden Beziehungen zwischen, unterschiedlichen Betonungen auf und oft widerstreitenden Interpretationen von diesen Aspekten des menschlichen Daseins“ entwickelt. Globalisierung ist vor diesem Hintergrund kein alleiniges Phänomen unserer heutigen Gesellschaft, sondern kann über viele Jahrhunderte hinweg in unterschiedlichen Formen beobachtet werden. Unsere heutige Form der Globalisierung enthält das, was Robertson „Glokalisierung“ nennt. Kurz gesagt, ist damit die widersprüchliche Einheit von Lokalem und Globalem gemeint. Das Lokale ist kein Gegenspieler des Globalen mehr, so wie es in vielen anderen Ansätzen der Globalisierungsdebatte gerne definiert wird, sondern ein Aspekt von Globalisierung. Zugleich wird so eine „methodisch-pragmatische Wende“ (Beck 1997) in der Globalisierungsforschung vollzogen, indem der Blick auf das Globale im Lokalen, Konkreten, im alltäglichen Leben gelenkt wird. Die zentrale Auseinandersetzung um globale Homogenisierung contra Heterogenisierung wird als überholt verabschiedet. Globalisierung kann ebensowenig mit homogenisierenden Trends gleichgesetzt werden wie Lokalität mit Widerstand gegen das hegemoniale Globale. Beide Strukturen durchdringen sich bzw. stehen zueinander in wechselseitiger Beziehung.

Insgesamt ist mit der Analyse globaler Kultur in dialektischen Prozesskategorien ein Perspektivenwechsel in der soziologischen Globalisierungsdiskussion eingeläutet worden, der sich von den „klassischen“ Ansätzen abgrenzt, in deren Analyse vor allem Kategorien geschlossener und begrenzter Räume von Nationalstaaten und ihren Nationalgesellschaften zu Grunde gelegt werden. Um also heute die Frage zu beantworten, was das Globale in unserem Leben - auf unser spezielles Thema fokussiert: in sozialen Lebenszusammenhängen von Jugendlichen und Medien - ausmacht, hilft das Denken in kulturell-homogenisierten Strukturen kaum mehr weiter. Der analytische Blick muss sich für zwischenkategoriale, hybride Lebensformen öffnen (vgl. Canclini 2001; Nederveen-Pieterse 1998), die im Kontext von Globalisierungsprozessen entstehen und die eine zunehmende Bedeutung in unserer Gesellschaft erlangen. 
Dass die Medien in kulturellen Globalisierungsprozessen eine besondere Rolle spielen und damit unser soziales Miteinander einschneidend beeinflussen, wird schon durch die mittlerweile gewohnte Live-Berichterstattung des Nachrichtensenders CNN von weit entfernten Krisenherden verdeutlicht, etwa vom Beschuss Bagdads durch amerikanische Bomber im Rahmen des ersten Golfkriegs, um einen der Schlüsselmomente globaler Kommunikation im Fernsehen zu nennen. Bereits in den 1960er Jahren hat der kanadische Kommunikationswissenschaftler Marshall McLuhan die kulturellen wie sozialen Effekte des Verhältnisses zwischen Medien und Globalisierung analysiert, zugespitzt in der Idee des global village (vgl. etwa McLuhan 1964). McLuhan stellt dar, wie insbesondere das elektronische Medium Fernsehen eine neue historische Epoche der Menschheitsgeschichte kennzeichnet, durch die die Gutenberg-Galaxis - die Ära des Buchdrucks - abgelöst wird. Die Schnelligkeit elektronisch basierter Kommunikations- und Transportmedien führe einerseits zur technischen Erweiterung unseres Bewusstseins, andererseits zu dem strukturellen Effekt der „Implosion“, d.h. zur raumzeitlichen Komprimierung der Welt und des menschlichen Erfahrungsraumes. Auf diese Weise werde es nun möglich, dass wir weit entfernte Ereignisse an jedem Ort der Welt simultan miterleben können; prinzipiell könne jeder Mensch mit allen anderen Kontakt aufnehmen. McLuhans Überlegungen müssen vor dem Hintergrund „seiner Zeit“ verstanden werden, im Fernsehbereich gekennzeichnet durch das Aufkommen der ersten Satellitentechnologien. Erst mit ihrer Hilfe wurde es möglich, Bilder und Nachrichten von einem Punkt der Erde jetzt auch weltweit zu verbreiten, so dass wir, so McLuhan, das Leben bisher fremder Menschen, Kulturen und Gesellschaften in unser aller Leben miterfahren und -einbeziehen können. Die neuen technischen Möglichkeiten, über die die Gesellschaft in Form der elektronischen Medien verfugt, errichten ein internationales Netzwerk der Kommunikation, das mit dem zentralen Nervensystem des Menschen vergleichbar sei: Internationale Medien-Events und weltweit populäre Fernsehserien - die Mondlandung oder der Kennedy-Mord in den 1960er Jahren, Dallas in den 1980er Jahren - produzieren eine gemeinsame Kommunikationssphäre, die über Kontinente hinweg besteht und uns dazu befähigt, die Welt als Ganze sinnlich zu erfassen.

McLuhans Verdienst ist es, dass er in seinen Analysen über die Inhalte der Medien hinaus geht und erstmals den kommunikationswissenschaftlichen Blick auf die kulturelle Bedeutung des Verhältnisses von Mensch und Medien gelenkt hat. Sein Ansatz kann dort als übertrieben oder unzutreffend beurteilt werden, wo er das Massenmedium Fernsehen in erster Linie als Motor kultureller Homogenisierung darstellt, eine These, die so kaum zu belegen ist. Nicht zuletzt muss er sich auch den Vorwurf des technologischen Determinismus gefallen lassen (vgl. Burnett/Marshall 2003), weil er die gesellschaftliche Entwicklung eindimensional auf die Medien zurückführt; der Mensch als aktiver Produzent seiner gesellschaftlichen und kulturellen Wirklichkeit wird jedoch zu wenig berücksichtigt. Dennoch: Bis heute hat sein Begriff des global village für die weite- 
re Erforschung des Zusammenhangs von Globalisierung und Medien wichtige Anstöße gegeben (vgl. Robertson 1992).

Während in den 1970er Jahren die Medien zunächst in ihrer Rolle als monopolisierende wie manipulisierende Akteure der Gesellschaft betont wurden und Herbert Schillers bekannte These vom cultural imperialism durch vor allem amerikanische bzw. verwestlichte Massenmedien im Vordergrund der Debatte stand (vgl. Schiller 1970), hat sich das zentrale Paradigma kultureller Homogenisierung spätestens seit Ende der 1980er Jahren gewandelt. Vor dem Hintergrund der jüngeren Gobalisierungsdebatte werden auch im kommunikationswissenschaftlichen Kontext die „alten“ Vorstellung über globalisierender Auswirkungen elektronischer Medien von differenzierteren Theorien und Konzepten zunehmend relativiert. Das „globale Homogenisierungs-Szenario“ (Hannerz 1991) des Kulturimperialismus-Ansatzes, das davon ausgeht, es setze sich lediglich eine Kultur durch - und zwar die westliche -, die unseren Lebensstil bestimmt und zahlreiche andere, verschiedene Kulturen verdrängt oder auslöscht, wird als zu pessimistisch oder falsch entlarvt. Um den Einfluss der Globalisierung auf unsere globale Kultur adäquat deuten zu können, wird auch für das Medienthema eine eher dialektisch angelegte Perspektive vorgeschlagen, die sich nicht mehr von dem strukturellen Muster Zentrum-Peripherie leiten lässt, sondern von Dezentralisierung (vgl. Tomlinson 2002).

In diese Richtung argumentiert auch Manuel Castells (2001), der unsere gegenwärtige Gesellschaft zunehmend in Netzwerken organisiert sieht. Zwar habe es Netzwerke als Form sozialer Organisation bereits zu anderen Zeit gegeben, das neue informationstechnologische Paradigma schaffe aber nun die materielle Basis dafür, dass diese Form eine durchdringende gesellschaftsstrukturelle Bedeutung gewinnt. Nach Einschätzung von Castells leben wir in einer Netzwerkgesellschaft, in der die herrschenden Funktionen in Netzwerken organisiert sind, die dem Raum der Ströme angehören - anstelle eines Raumes der Orte -, der sie weltweit miteinander verbindet „und zugleich die untergeordneten Funktionen und Menschen in vielfältige Räume von Orten fragmentiert, die aus immer stärker segregierten und abgekoppelten Örtlichkeiten bestehen“ (ebd., S. 535). Die materiellen Grundlagen dieser Ströme befinden sich auf drei Ebenen (ebd., S. 466ff.): erstens der elektronische Kreislauf der Vermittlung (Telekommunikation, mikroelektronische Geräte etc.); zweitens Knoten und Zentren, die sich innerhalb eines Netzwerkes - z.B. die Global City (vgl. auch Sassen 1991) - als spezifische Orte darstellen; drittens die räumliche Organisation der herrschenden Führungseliten. Die herrschenden Funktionen in unserer Gesellschaft werden geformt durch Inklusion in und Exklusion aus Netzwerken sowie die Gestalt der Beziehungen zwischen Netzwerken, die durch Informationstechnologien rasend schnell in Gang gesetzt werden. Das Internet spielt in Castells Trilogie des Informationszeitalters eine entscheidende Rolle, jedoch nicht nur als Technologie, sondern im Sinne der spezifischen Netzwerklogik, auf der gesellschaftliche Globalisierung und globale Kommunikation beruhen. 
Festzuhalten ist: Die Gestalt globaler Kultur scheint sich heute in Form netzwerkartiger, widerspruchsvoller, zwischenkategorialer, glokaler, fluider wie pluri-örtlicher Strukturen widerzuspiegeln. In der Erforschung von Globalisierungsprozessen ist ein Paradigmenwechsel auszumachen. Der nationale Blick auf die gesellschaftliche Entwicklung wird in Frage gestellt und kritisiert, weil er in homogenen Erklärungseinheiten des Sozialen verhaftet bleibt. Zudem ist er zu unpräzise, um die zunehmend zu beobachtenden transnationalen sozialen Verbindungen auf den Begriff zu bringen. Wenngleich derzeit Uneinigkeit vorherrscht, wie das neue Paradigma genau zu denken ist, ob eher in Termini „moderner“ (vgl. Beck 2002; Giddens 1990) oder post-moderner Globalisierung (vgl. Castells 2001; Appadurai 1998), besteht in der Debatte kaum Zweifel darüber, dass eine Globalisierung unserer Lebenswelten stattfindet. Gleichwohl befindet sich deren sozialwissenschaftliche Deutung erst am Anfang. Unbestritten ist auch, dass die elektronische Kommunikation einen entscheidenden Einfluss auf die komplexen Globalisierungsprozesse ausübt.

\section{Jugend(kulturen) und Globalisierung}

Welche Auswirkungen hat die Globalisierung auf das Jugendalter? Zwar können über das gesamte 20. Jahrhundert Jugendkulturen beobachtet werden, die nicht auf begrenzte nationale Räume beschränkt sind - etwa die Jazz- und Swingbewegung in den 1920er Jahren oder die Teenager und Halbstarken in den 1950er Jahren. Aber der Effekt von Globalisierungsprozessen auf Jugendkulturen zeigt sich erst voll mit dem Beginn der weltweiten Verbreitung und Vermarktung von Musik- und Medienprodukten seit etwa den 1970er Jahren (vgl. Vollbrecht 2002).

Deshalb überrascht es, dass diese Zusammenhänge bisher kaum Eingang in die Jugendforschung in Deutschland gefunden haben. Eine der wenigen Ausnahmen stellt der Beitrag von Roland Roth „Globalisierungsprozesse und Jugendkulturen“ dar. Roth stellt die These auf, dass Jugendkulturen durch Globalisierung geprägt oder verändert werden. Er unterscheidet drei gegensätzliche jugendkulturelle Reaktionsformen auf Globalisierungsprozesse (Roth 2002, S. 24 ff.):

1) „Jugendkulturen als Vorreiter von Globalisierungsprozessen“: Im Produzieren und Ausleben immer neuer Lebensstile und Moden wird eine „proaktive Grundströmung“ der Jugendlichen entdeckt. Diese Gruppe ist aber lediglich Erfüllungsgehilfe eines ökonomischen Globalisierungsprozesses, der sich in dem „Projekt einer weltweiten kulturellen Homogenisierung ausdrückt (Stichwort: "McDonaldisierung“). Die globale Kommerzialisierung werde den Jugendkulturen von mächtigen Konzernen ,aufgeherrscht“.

2) „Identitätsorientierter Widerstand und Rückzugsbewegungen“: Diese Merkmale zeigen sich in vielfältigen „lokalen Gegenkulturen“, die religiös, regionalistisch, nationalistisch, rechtsextrem oder ökologisch motiviert sein können. Ihr „Rückzug in Gemeinschaften“ sei eine Reaktion auf den staatlichen Kontrollverlust, der an die 
Globalisierung vermeintlich oder tatsächlich gekoppelt ist.

Von diesen beiden Strömungen grenzt Roth

3) „globalisierungskritische Milieus und Bewegungen“ ab. In ihnen sieht er einen „progressiven globalisierungskritischen Gegenpol“ sowohl zur "proaktiven Strömung“ als auch zu den auf nationale wie regionale Schließung setzenden Rückzugsbewegungen. Ihr Ziel sei konstruktiv, weil sie sich für eine „sozial gerechte, demokratische Gestaltung der Globalisierungsprozesse durch die Rückgewinnung politischer Gestaltungsspielräume“ (ebd., S. 27) im Rahmen von konsumkritischen Initiativen, Boykottaktionen gegen Markenhersteller oder in globalisierungsktischen Netzwerken (Beispiel „Attac“) engagieren.

Gegen Roths’ Argumentation lassen sich mindestens zwei kritische Punkte Vorbringen: Erstens betont er die Negativfolgen der Globalisierung über: Nicht abzustreiten ist, dass Ungleichheitsdynamik und soziale Exklusion sowie Abstraktion und Gestaltungsmangel und Konflikthaftigkeit zu den globalen Herausforderungen gehören. Warum aber ausschließlich diese eine Seite der Medaille hervorgehoben wird, bleibt in Roths Argumentation unklar. Angesichts globaler kultureller Verhältnisse hätte er zugleich auf neu entstehende Elemente des „Gemeinsamen“ hinweisen müssen, die durch Globalisierung entstehen. Zu denken ist etwa an die Ansätze einer globalen, vernetzten Öffentlichkeit - einer „Global Sphere of Mediation“ (vgl. Volkmer 2003) -, wie sie etwa in dem Nachrichtenformat „World Report“ bei CNN zum Ausdruck kommt (vgl. auch Volkmer 1999). Roth muss auch dort widersprochen werden, wo er Globalisierung alleine im Sinne von Homogenisierung definiert: Auf Grund von ökonomischen Globalisierungstendenzen wird für ihn eine zunehmend kulturelle Vereinheitlichung erzeugt. Deshalb leben wir in einer globalen Welt, die eine Waren- und Konsumwelt ist. Auf diese Weise zeigt Roths Ansatz eine deutliche Nähe zum Wallerstein'schen Ansatz einer kapitalistischen Weltökonomie. Zwar erwähnt Roth die lokalen jugendkulturellen Strömungen. Dieses Lokale wird aber entweder als ausschließlich lokale Gegen-Reaktion zum Globalen verstanden oder als lediglich „lokaler Akzent“, der gegenüber den Auswirkungen weltweiter Kommerzialisierung zweitrangig erscheint. Der Ansatz von Roth ist damit zu ungenau, um auch die Paradoxien kultureller Globalisierung zu erklären - die widersprüchliche Einheit von Globalisierung und Lokalisierung. Wenn sich also Globalisierung und Lokalisierung wechselseitig durchdringen, dies wird übersehen, kann Globalisierung auch eine „Produktion“ von Lokalisierung bedeuten. 


\section{Globalisierte Medienszenen von Jugendlichen: Zwei Beispiele}

Diese (neuen) Formen der Globalisierung können sich in den Medienszenen von Jugendlichen ausdrücken. Dieter Baacke (1988) nennt drei strukturelle Gemeinsamkeiten jugendlicher Medienszenen, die bei aller Unterschiedlichkeit festgemacht werden können: „Intensität“, „Ganzheit“ und „Subjektivität“:

„Intensität meint, dass die Rezipienten-Haltung einer distanzierten Beobachtung verlassen wird: Mediennutzung wird zum Medienereignis ... Ganzheit meint, dass die vorhandenen, öffentlichen Medien zu abstrakt sind, um die direkten lebensweltlichen Erfahrungen einzufangen und wiederzugeben. Jugend-Medien müssen lebensraum-bezogen, lokalorientiert und in ihren Nachrichten konkret sein. Gleichzeitig neigen Jugendliche stärker als andere Altersgruppen dazu, unterschiedliche Medien zu benutzen, um auch auf diese Weise ganzheitliche Intensitätserfahrungen möglich zu machen ... Subjektivität meint, dass Jugendliche sich sehr wohl ,vergemeinschaftet ‘ fühlen, also als Individuen, deren Lebensweg durch gesellschaftliche Kontrollinstanzen weitgehend strukturiert ist... Die Medien-Szenen erlauben ihnen, im Misstrauen gegen die etablierte Öffentlichkeit Momente ihrer eigenen Subjektivität zum Ausdruck gelangen zu lassen“ (Baacke 1988, S. 40).

Baacke berücksichtigt jedoch kaum die gesellschaftlichen Globalisierungsbedingungen. Auch sein Konzept muss deshalb überprüft, mit den Kennzeichen globalisierter Medienszenen abgeglichen und ergänzt werden. Dazu gehört vor allem, dass die globale (Medien-)Kultur dialektisch-prozesshaft zu verstehen ist. Was heißt dies konkret, wenn jugendliche Medienszenen untersucht werden? Insbesondere sind zwei strukturelle Unterschiede wichtig: erstens das Verhältnis von Globalität und Lokalität, zweitens die Entstehung neuer transnationaler sozialer Räume. An zwei Beispielen globalisierter Medienszenen von Jugendlichen soll dies im Folgenden verdeutlicht werden. Die Aufzählung könnte aber durch die Nennung weitere Szenen ergänzt werden.

\section{Glokale HipHop-Szene}

Jugendszenen messen ihrer Musik eine besondere Bedeutung bei. Über Musik können insbesondere Fragen der Identität und des Sinns vermittelt werden. Dies gilt auch für HipHop. Die Ausdrucksformen des HipHop gehen über Rap hinaus und umfassen auch Djing, Graffiti und Breakdance. Es handelt sich um eine „mehrdimensionale kulturelle Praxis“ (Androutsopoulos 2003a), in der Bild, Sound, Typografie, Körperbewegung und Sprache in Beziehung zueinander stehen und von den Akteuren kombiniert werden. Ausgehend von der afroamerikanischen Straßenkultur Anfang der 1970er Jahre in New York - verbunden mit Namen wie Grandmaster Flash oder Afrika Bambaataa und seiner Zulu-Nation - hat sich die HipHop-Kultur bis heute in verschiedenste „Spielarten“ ausdifferenziert. Selbst die deutsche HipHop-Szene kann auf eine fast 20jährige Geschichte zurückblicken, die Anfang der 1980 Jahre mit der Break- 
dance-Welle erwachte und mit den Fantastischen Vier Anfang der 1990er Jahre („Die da?") einen öffentlichen Durchbruch erlebte.

In Deutschland kann heute eine ausdifferenzierte HipHop-Kultur beobachtet werden. Globalisierung kommt in dieser jugendlichen Medienszene vor allem im Verhältnis von Globalem und Lokalem zum Ausdruck. Bereits ein kurzer Blick auf die deutschen, französischen oder amerikanischen Musik-Charts macht deutlich, dass HipHop kein „globales“ jugendkulturelles Phänomen darstellt, wenn darunter alleine Gleiches und Ununterscheidbares verstanden wird. HipHop stellt ein zugleich lokales Phänomen dar, das bei Jugendlichen in Frankfurt am Main oder Berlin-Kreuzberg sehr unterschiedliche Formationen aufweisen kann (vgl. Bennett 2003). Zwar können lokale Elemente der Popkultur auch in anderen Musikszenen beobachtet werden, die Beständigkeit und Wirksamkeit, mit der die lokale Aneignung der HipHop-Kultur erfolgt, hat aber besonders ausgeprägten Charakter. Die Gründe dafür können in der Erscheinung des HipHop selbst gesucht werden, und zwar, wie Androutsopoulos (2003a, S. 12) meint: erstens in der Zugänglichkeit der Kultur (geringe technische Hemmschwellen, keine formale Ausbildung notwendig), zweitens in ihrem performativen Charakter (Mitmachen, Dabeisein, aktive Beteiligung), drittens in der Arbeit am Style (HipHop bietet Raum für kreatives Umgestalten, etwa durch das Zitieren von anderen Musikstücken oder -Stilen) und viertens im Prinzip des Wettbewerbs (ständiger und offener Wettstreit miteinander). Vor allem der Rap wird von den Akteuren dazu benutzt - dieser Aspekt kommt hinzu -, sich mit den lokalen kulturellen Erfahrungen auseinander zu setzen. Von der afroamerikanischen Ur-Form hat sich zwar der HipHop in Deutschland weit entfernt, dennoch sind beide Varianten Bestandteile eines Netzwerks kultureller Bezüge, in dem sich die jugendlichen Szenenmitglieder immer wieder neu verorten. Das Sampling (Modifizieren, „Wiederverwenden“, Verfremden) von Musikstücken durch Rap-Gruppen bietet dafür das geeignete Instrument an, um in deren Tracks - so Mikos (2003) - ein „intertextuelles Referenzsystem“ zu schaffen, das mittlerweile ständig auf den „eigenen kulturellen Kontext" hinweist.

Hier kann jedoch immer weniger von einem eigenen deutschen kulturellen Kontext gesprochen werden. Denn selbst wenn die Fantastischen Vier oder Fettes Brot in deutscher Sprache rappen und Die Firma die 40. Symphonie von Mozart sampelt, bezieht sich doch die HipHop-Szene in Deutschland auf ganz unterschiedliche kulturelle Elemente, die auch Gegensätze zum Ausdruck bringen können. Was das bedeuten kann, zeigt Bennett (2003, S. 34f.) anhand von eigenen Erfahrungen mit deutsch-türkischen HipHoppern in Frankfurt. Er ergänzt seine Darstellung durch Interviewaussagen, etwa vom Leiter eines kleinen, unabhängigen Plattenlabels im Frankfurter Westen, das sich auf türkische Rap-Musik spezialisiert hat:

„Also, musikalisch gesehen versuchen wir traditionelle türkische Melodien mit Rap-Rhythmen zu kombinieren. Die Kids machen das schon eine ganze Zeit 
lang ... Man kann türkische Musikkassetten überall in der Stadt in türkischen Läden kaufen, und sie experimentieren mit dieser Musik, sampeln sie, mischen sie mit anderen Sachen und rappen dazu ... Wir versuchen dieses türkische Rap-Ding etwas auszubauen, indem wir es vertreiben ... Wenn ich ihnen sagen soll, warum wir so etwas machen, naja, es ist eigentlich aus Stolz. Die Sache mit viel von diesem deutschen Rap ist, dass es da diese farbigen Typen gibt, die sagen, schaut her, wir sind wie ihr, wir sind deutsch. Aber so fühle ich mich nicht, und ich habe mich noch nie so gefühlt, ich bin Türke und unheimlich stolz darauf“.

Für Andy Bennett wird in dieser Aussage deutlich, dass insbesondere HipHop von Jugendlichen ethnischer Minderheitengruppen dafür genutzt wird, um sich im lokalen Raum (hier: in Frankfurt) kulturell auszudrücken. Allerdings greift die LokalisierungsThese dann zu kurz, wenn sie das Lokale vor allem als Gegensatz zum Globalen festgelegt: Dann ist Lokalisierung eine Reaktion auf Globalisierung. In den globalisierten Medienszenen - so möchte ich stattdessen argumentieren - stellt das Lokale ein Aspekt des Globalen dar. Das heißt z.B. für die türkische HipHop-Jugend in Frankfurt: als globalisierte Medienszene nimmt sie zwar die vereinheitlichten Symbole globaler Kultur in Rap, Djing, Graffiti und Breakdance auf und verarbeitet sie, dies stellt aber gerade keinen Gegensatz zur Behauptung ihrer lokalen Identität dar, weil sie die „allgemeinen“ Symbole des HipHops in ihrem eigenen kulturellen Kontext spezifisch interpretieren. Diese Dialektik von Globalem und Lokalem erklärt auch, warum urbane afroamerikanische Symbole und türkische arabeske Musik für die Jugendlichen keinen Widerspruch darstellen (vgl. Kaya 2003). Auf diese Weise „erfinden“ sich die glokalen HipHop-Szenen - jenseits eines „Entweder-oder“ - als etwas neuartiges „Drittes“, als Cross-OverKulturen.

Transnationale soziale Räume von Migrantenjugendlichen im Internet

Ein wesentliches Ergebnis der Globalisierungsforschung, die in den letzten Jahren auch nach empirisch belegbaren Zeichen globaler Kultur sucht, ist die Entdeckung transnationaler sozialer Räume. Damit werden Formen sozialer Lebensund Handlungszusammenhänge bezeichnet, die regelmäßigen und anhaltenden sozialen Kontakt über nationalstaatliche Grenzen hinweg erfordern (vgl. Portes/Guarnizo/Landolt 1999). Raum ist dabei als Metapher zu verstehen, die widersprüchlich benutzt wird, denn das bedeutendste Merkmal dieser Räume ist, dass sie Entfernungen aufheben. „Transnational“ bedeutet - so Beck (1997, S. 63f.): „Es entstehen Lebens- und Handlungsformen, deren innere Logik sich aus dem Erfindungsreichtum erklärt, mit denen Menschen ,entfernungslose‘ soziale Lebenswelten und Handlungszusammenhänge errichten und aufrechterhalten“. Sie gewinnen heute immer mehr an Bedeutung für die soziale Wirklichkeit, vor allem in Verbindung und wechselseitigem Verhältnis von Globalisierung und neuen Kommunikations- und Informationstechnologien.

Pries (2001, 2002) zeigt dies am Beispiel der Migrationsforschung. Während traditionelle Vorstellungen von (Arbeits-)Migration, die internationale Wande- 
rungsdynamik lediglich in den Kategorien eines definitiven Wechsels aus einem Herkunftsland bzw. einer spezifischen Herkunftsregion in eine neue Ankunftsgesellschaft zu erklären und verstehen versucht haben (Emigration/Immigration), erhält im Zusammenhang mit Globalisierungsprozessen ein neuer Typ internationaler Migration zunehmend Bedeutung, der nicht mehr allein mit den traditionellen Denkansätzen erklärt werden kann. Es geht um diejenigen Migranten, für die der Wechsel zwischen verschiedenen Lebensorten in unterschiedlichen Ländern kein einmaliger Vorgang ist, sondern ein dauerhafter Zustand und zur Normalität wird, indem sich ihr gesamter Lebensraum „pluri-lokal über Ländergrenzen hinweg zwischen verschiedenen Orten“ verteilt (Pries 2001, S. 16). Es entstehen transnationale Sozialräume, die etwas Drittes darstellen, ein hybrider Lebenszusammenhang aus Elementen der Herkunfts- und Ankunftsregionen (vgl. im Hinblick auf die empirische Erforschung etwa Mandaville 2001; Smith 2001). Diese Prozesse finden in den letzten Jahren auch Widerhall in der Debatte um internationale bzw. transnationale Kommunikation (vgl. dazu etwa den Sammelband von Hepp/ Löffelholz 2002). Dabei wird in den Cultural Studies vor allem der Begriff der Diaspora diskutiert, verstanden als „gleichzeitige Zuordnung zu mehreren Orten, als besonderer eigengesetzlicher Lebensraum oder als Raum, der sich zwischen Territorien und Identitäten erstreckt“ (Bromley 2001, S. 796). Im Mittelpunkt steht das Verständnis der Produktion von „hybriden“ Identitäten und Kulturen „durch die gleichzeitigen Aktivitäten von Aufrechterhaltung und Aushandlung der Heimatkultur und der neuen Zielkultur“ (Cunningham/Nguyen 1998, S. 2).

In solchen transnationalen Formationen können Medien eine zentrale Rolle spielen, weil sie es sind, die die sozialen Aushandlungsprozesse unterstützen und beeinflussen. Silverstone (2002) bringt dies auf den Punkt, wenn er betont: „Die Medien stellen hierfür Mittel zur Verfügung; sowohl die Medien, die von den Gemeinschaften produziert werden, als auch die Medien, die sie konsumieren, die Medien ihrer eigenen ,Heimat'kultur und die ihrer ,Gastkultur. Aus diesen Prozessen entsteht etwas Neues: ein kleines Kosmopolitentum, eine veränderliche Hybridität, die in alten wie neuen Medien reflektiert und ausgedrückt wird“ (Silverstone 2002, S. 743). Dieser Zusammenhang ist bereits für unterschiedliche Gruppen untersucht worden. Dazu zählen vietnamesische diasporische Kulturen in Australien und Kalifornien (Cunningham/Nguyen 1999), die iranische Exilkultur in Los Angeles (Naficy 1993), Iraner in London (Sreberny, im Erscheinen) oder die südasiatische Diasporakultur in West-London (Gillespie 1995).

Die Gestalt transnationaler sozialer Verbindungen ist allerdings immer weniger durch unmittelbare Migrationserfahrungen und abgesteckte territoriale Grenzen festgelegt. Für die Entstehung gegenwärtiger transnationaler sozialer Räume ist vielmehr entscheidend, dass zwischen den beteiligten Personen eine bestimmte Form gemeinsam geteilter Bewusstheit besteht, eine „imaginary coherence“ (Hall 1990), die den geeigneten Rahmen für wichtige Identitätsbildungsprozesse darstellt. Robert Cohen (1996, S. 516) merkt deshalb an: „In the 
age of cyberspace, a diaspora can, to some degree, be held together or re-created through the mind, through cultural artefacts and through a shared imagination“.

Für unser Thema bleibt in der Erforschung transnationaler sozialer Räume zunächst zweierlei offen:

- Erstens gibt es kaum Hinweise auf jugendspezifische Aushandlungsformen in diesen Räumen. Gerade weil es so ist, dass Jugendliche heute verstärkt selbst darüber entscheiden (müssen), welche symbolischen Gebrauchsmuster sie für sich erwerben und wie sie diese in ihr Leben integrieren (vgl. Baacke 2000), ist zu fragen, was dies in transnationalen Lebenszusammenhängen bedeutet. Welche Anhaltspunkte gibt es überhaupt dafür, ob und wie sich transnationale soziale Räume von Jugendlichen entwickeln?

- Zweitens bleibt noch weitgehend offen, welche Bedeutung die elektronischen Medien, vor allem: die neuen Informations- und Kommunikationstechnologien, bei der Konstituierung von solchen Räumen von Jugendlichen genau spielen. Verschiedene Einschätzungen weisen zwar auf die zentrale Rolle des Internets hin. So geht etwa Silverstone (2002) davon aus, dass die sozialen Netzwerke von Migranten - Punjabis in Southall, marokkanische Juden in Bordeaux oder Türken in Berlin - zunehmend mit Hilfe der Neuen Medien funktionieren (vgl. auch Pries 1998). Über diese allgemeinen Einschätzungen hinaus, finden sich aber über die spezifische Bedeutung der Internetkommunikation in transnationalen Lebenszusammenhängen (von Jugendlichen) kaum oder gar keine Untersuchungen.

Diese Perspektiven sind - dies muss betont werden - nicht mit Untersuchungsansätzen zu verwechseln, die alleine den Prozess der interkulturellen Kommunikation zwischen Menschen oder Gruppen unterschiedlicher Nationen bzw. Kulturen im Blick haben. Hier soll vielmehr davon ausgegangen werden, dass „neue Formen der Grenzziehung“ (Pries 2001) entstehen, die quer zu begrenzten nationalen und kulturellen Einheiten liegen. Aufgabe der Erforschung von transnationalen Sozialräumen von Jugendlichen ist es, neue Mischverhältnisse, Zwischenkategoriales, Ambivalentes, das „zugleich Hier-unddort-Sein“ (Beck 1997) in elektronischen Kommunikationszusammenhängen zu erschließen.

Auf der Suche nach solchen sozialen Formationen wird deutlich: Transnationale soziale Räume von Jugendlichen lassen sich zunehmend im Internet entdecken, etwa in den Kommunikations- und Interaktionsräumen von Migrantenjugendlichen. Ein Beispiel ist VAYBEE.DE („Wow“), das auch als „Ethnoportal“ oder „Multikulti-Portal“ (Azrak 2002) für deutsch-türkische Jugendliche im Netz bezeichnet werden kann. Neben VAYBEE.DE - weitere Beispiele sind turkdunya.de oder bizimalem.de - haben sich in den letzten Jahren auch für polnische (www.polonium.de) und griechische Migranten (z.B. www.alphanea.de) spezifische Internetangebote entwickelt (vgl. auch Dette 2003; Stegers 2003). Sie bieten einerseits eigene oder übernommene redaktionelle Inhalte an, andererseits stellen sie elektronische Foren (asynchron) und Chats (synchron) zum kommunikativen Austausch der Nutzer zu Verfügung. 
Mit ca. 250.000 registrierten Nutzern stellt VAYBEE.DE die vermutlich meist genutzte Website dieser Art bei türkischen Jugendlichen dar (vgl. Senay 2003). Der türkische Journalist Attila Azrak beschreibt ihr Angebot wie folgt:

„Auf der Eingangsseite findet sich alles, was den Jugendlichen von heute interessiert. Sprachlich nah an der Zielgruppe gehalten, wird die türkische Surfergemeinde durch Angebote wie News-Häppchen, Lifestyle-Meldungen und Online-Shopping für Markenwaren angesprochen. VAYBEE.DE versteht sich als deutsch- und türkischsprachiges Portal für türkische Jugendliche, die sich untereinander auf Deutsch und Türkisch unterhalten. Dies belegt auch ein geschätzter deutscher Useranteil von fünf Prozent. (...) Neben den üblichen Angeboten wie einem Veranstaltungskalender (hauptsächlich Tanzveranstaltungen) und einer auf die besonderen Bedürfnisse der jungen Migrantengeneration zugeschnittenen Jobbörse, sind auch spezielle Angebote wie türkische Behördenratgeber in die Site integriert. VAYBEE.DE lockt türkische Surfer mit zusätzlichen Angeboten wie einem Shop für CDs oder einem Mobilportal für Klingeltöne und Logos für Mobiltelefone“ (Azrak 2002, S. 10).

Betreiber der Website ist die VAYBEE AG, die von den drei türkischen Brüdern Hasim, Tamer und Akgün Kulmac im Mai 2000 gegründet wurde. Da es sich um ein kommerzielles Angebot handelt, verwundert es nicht, dass neben - nach wie vor frei zugänglichen - „Grundfunktionen“ seit Ende 2002 zunehmend auch kostenpflichtige „Premiumservices“, beispielsweise die nach eigenen Angaben erste türkische Partnerbörse Europas im Internet, angeboten werden. Diese verstärkte Kommerzialisierung des Angebotes hat zwar zu einigem „Ärger“ in der „Community“ geführt, was durch zahlreiche Postings in den elektronischen Foren belegt werden kann. Bisher hat sich dieser Unmut aber offenbar nicht in einer geringeren Akzeptanz des Gesamtangebotes niedergeschlagen. Tamer Kulmac, Mitbegründer und Geschäftsführer weist stattdessen auf die große Beliebtheit des Kommunikationsbereichs hin: „Man kann nicht sagen, dass er (der User, K.U.H) wegen der Nachrichten auf unsere Site kommt. Hauptsächlich werden unsere interaktiven Angebote wie Email oder Homepages, Diskussionen und Chats genutzt. Hier kommunizieren die User untereinander und das sind eigentlich die ,Community-Tools‘. Wir haben sowohl Informationen aus der Türkei, als auch türkische Themen aus Deutschland und Europa in unseren Angebot“ (Interview mit Tamer Kulmac 2002, S. 11).

In den Sozialwissenschaften sind solche sozialen Phänomene im Internet bisher vor allem mit der Bildung „virtueller Gemeinschaften“ bzw. „Online-Communities“ in Zusammenhang gebracht worden. Noch wird aber nach genauen Hinweisen dafür gesucht, wie in den elektronischen Netzwerken „Gemeinschaft“ gefunden wird und welche kulturellen Folgen dies hat (vgl. Castells 2001; Marotzki 2003). Das mittlerweile auch in einer breiteren Öffentlichkeit zu beobachtende Interesse an der Untersuchung von Internet-Communities lässt sich teils mit der Vorstellung erklären, dass in den virtuellen Foren ein durch gegenseitige Hilfe und soziale Nähe gekennzeichnetes soziales Miteinander vorherrscht, das in der modernen Gesellschaft schon längst verloren geglaubt wur- 
de. In diesem Sinne muss auch Rheingold (1993) verstanden werden, der als einer der entschiedensten Verfechter für die Entstehung einer neuen Form von Gemeinschaft im Netz gilt. Virtuelle Gemeinschaften sind für ihn die Folge eines wachsenden Bedürfnisses nach Gemeinschaft, das die Menschen weltweit entwickeln, weil in der „wirklichen Welt“ die Räume für ungezwungenes soziales Miteinander immer mehr verschwinden. Die Online Communities sieht er als Brücke zu fremden Kulturen an, die jetzt nicht mehr unbedingt von Angesicht zu Angesicht besucht werden müssen, damit man sie kennen lernt. Auch eine virtuelle Begegnung könne dies jetzt ermöglichen, wenngleich Face-to-face-Treffen dadurch nicht ersetzt würden.

Folgt man diesem Gedanken weiter, würde dies bedeuten, dass durch die virtuell geknüpften Bekanntschaften und die spezifische Art und Weise, in der man sich über computervermittelte Kommunikation (CvK) miteinander austauscht, völlig neue Erfahrungshorizonte erschlossen werden könnten, die sich von den bisherigen Wirklichkeitserfahrungen - medial vor allem durch Fernsehen und Telefon beeinflusst - unterscheiden. Rheingold (1993) definiert virtuelle Gemeinschaften als elektronische Netzwerke interaktiver Kommunikation, die von einem gemeinsam geteilten Interesse oder einem gemeinsamen Zweck bestimmt werden. Dabei kann auch die Kommunikation selbst zum Ziel werden. Deutlich wird insgesamt, dass zahlreiche Thesen zur Entstehung von Online-Communities ein häufig idyllisches Bild von Gemeinschaft als dichte, abgegrenzte und dorfähnliche Formen des sozialen Miteinanders vertreten, geprägt durch Nähe, Untersützung und Zugehörigkeit. Die Existenz dieser sozialen Formationen ist aber wohl selbst für die Agrargesellschaft zu bezweifeln. Nicht zuletzt spiegelt sich auf diese Weise das Verständnis klassischer soziologischer Theorien wider - verbunden etwa mit den Arbeiten von Tönnies, Parsons oder Dürkheim -, die das soziale Miteinander mit den Kategorien Gemeinschaft bzw. Gesellschaft zu beschreiben versuchen. Es bleibt aber festzuhalten: Die Verwendung des traditionellen Gemeinschaftsbegriffs für soziale Prozesse im Netz ist kritisch zu hinterfragen, weil er ortsgebundene bzw. nationalstaatliche bzw. kulturell fest abgesteckte Räume betont, die sich aber auf Grund von Globalisierungsprozessen heute immer mehr entgrenzt darstellen.

Dem Umstand, dass der Begriff der Gemeinschaft für die Kennzeichnung von Online-Communities problematisch geworden ist, wird in der Internetforschung heute vor allem mit dem Netzwerkkonzept begegnet. So kritisiert der kanadische Soziologe Barry Wellman in einer Reihe von theoretischen und empirischen Arbeiten zur Entwicklung von virtuellen Gemeinschaften insbesondere die Gruppensoziologie und regt an, Gemeinschaft nicht mehr als hierarchisch strukturierte, relativ homogene und dicht verbundene Gruppe zu definieren, sondern besser als soziales Netzwerk interpersoneller Beziehungen, das Soziabilität, Unterstützung, Information und ein Gefühl für Zugehörigkeit sowie soziale Identität liefert (vgl. etwa Wellman/Gulia 1999). Gegenüber den Überlegungen in der Gruppensoziologie sind solche Netzwerkkonzepte weit offener und ermöglichen es, sowohl die heterogene Teilnehmerstruktur in internet- 
basierten Diskussionsgruppen als auch die spezifischen sozialen Verbindungen in ihnen adäquater zu erfassen.

Mittlerweile ist bekannt, dass die Kommunikationsforen im Netz auch jugendkulturell angeeignet werden (vgl. Androutsopoulos 2003, Vogelgesang 2000). Zu nennen ist etwa die Szene der Globalisierungskritiker, für die das Netz die Grundlage für politische Meinungsbildung darstellt (vgl. Deutsche Shell 2002). Unerledigt ist allerdings die Entstehung von transnationalen sozialen Lebenszusammenhängen von Jugendlichen im Internet. Dabei geraten die jungen deutsch-türkischen Migranten in den Blick, deren Medienwelt bisher kaum untersucht worden ist (vgl. Schulte 2003). Eine genaue Aussage über deren Internetnutzung trifft selbst die von der Gesellschaft für Konsumforschung (GfK) im Auftrag des Bundespresseamtes 2001 vorgelegte Studie über die Mediennutzung und Integrationsbereitschaft der Migranten aus der Türkei nicht (vgl. Weiß/Trebbe 2001). Aussagen werden hier vor allem über die traditionellen Medien Fernsehen, Radio und Print getroffen.

Wie die Beliebtheit von VAYBEE.DE belegt, gewinnt aber insbesondere das Internet bei den jüngeren Deutsch-Türken zunehmend an Bedeutung. Im allgemeinen Sinne ist ihre Mediennutzung nicht auf „türkische“ Medien beschränkt. So hat die bereits genannte GfK-Untersuchung herausgefunden, dass in der türkischen Bevölkerung in Deutschland diejenige Gruppe am größten ist, die sowohl türkische als auch deutsche Medien nutzt. Jeder zweite Türke ist in seiner Mediennutzung „zweisprachig“. Am seltensten ist die ausschließliche Nutzung türkischsprachiger Medien. Deutlich größer ist dagegen die Gruppe der in Deutschland lebenden Türken, die ausschließlich deutschsprachige Medien nutzt (ebd.). Freilich unterscheiden sich die Nutzungsfunktionen des Internetangebotes von den traditionellen Medien. Neben „schnellen“ Informationen über Politik, Kultur etc., die auch über entsprechende Radioprogramme (etwa www.multikulti.de) oder das Fernsehen geliefert werden können, ist vor allem das Kommunikationsangebot in Foren und Chats zu nennen, das bei den Jugendlichen besonders beliebt ist.

Die Medienszene von deutsch-türkischen Migrantenjugendlichen im Netz ist transnational, weil sie sich über kommunikative Aushandlungsprozesse zwischen Herkunftsund Ankunftskultur konstituiert. Zur präzisen Charakterisierung dieser sozialen Formation scheint mir der Begriff „transnationaler sozialer Raum“ besser geeignet zu sein als die Bezeichnung „ethnische Online-Gemeinschaften“, die Androutsopoulos und Hinnenkamp (2001) zur Kennzeichnung des sozialen und kommunikativen Miteinanders von Migranten in Chats, Newsgroups und Ethnoportalen wählen. Als Ergebnis einer sprachwissenschaftlichen Untersuchung der Chats \#hellas und \#turks stellen sie fest: „Die Zugehörigkeit zur ethnischen Gruppe bzw. die Beherrschung der Sprache ist Teilnahmebedingung, der Status der ethnischen Minderheit sowie der Bezug zur Kultur des Herkunftslandes sind konstitutiv für das Selbstverständnis und die Thematik der Online-Interaktion“ (Androutsopoulos/Hinnenkamp 2001, S. 3). Diesen Überlegungen muss nicht grundsätzlich widersprochen werden, aber sie 
sind zu ungenau, wie die Analyse des Verhältnisses von „Globalisierung“ und „Jugendkulturen“ gezeigt hat. Zweifel kommen vor allem deshalb auf, weil die Autoren die Funktionen des Medienhandelns der Migrantenjugendlichen zu einseitig aus der Hinwendung zur - homogenen - Herkunftskultur heraus erklären. Demgegenüber legt die Nutzung der Kommunikationsforen von VAYBEE.DE - um nur ein Beispiel eines Ethnoportals zu nennen - eine komplexere Deutung nahe. Insbesondere scheinen es die Möglichkeiten der (anonymen) Internetkommunikation zu sein, die es für die Jugendlichen attraktiv machen, sich in den virtuellen Foren - jenseits von teils sehr groß erlebten sozialen Zwängen in vielen Migrantenfamilien - offener über auch prekäre kulturelle, soziale oder politische Themen zu unterhalten (Islam und Sexualität, allein erziehende türkische Mütter oder Beitritt der Türkei zur EU etc.). Dass dieses Angebot besonders gerne von deutsch-türkischen Mädchen/Frauen genutzt wird, sei hier nur am Rande bemerkt. Es liegt nahe, diese transnationale Kommunikationswelt dann als Bedingungsgefüge zu verstehen, das es Migrantenjugendlichen ermöglicht, eine „hybride“ Identität auszuhandeln, die gerade nicht allein auf die Herkunftskultur zu reduzieren ist. Die globalisierte Medienszene der Migrantenjugendlichen im Netz bietet den geeigneten „virtuellen“ Raum für diese Aushandlungsprozesse.

\section{Ausblick}

Am Beispiel der „glokalen HipHop-Szene“ und den „transnationalen sozialen Räumen von Migrantenjugendlichen im Internet“ sollte deutlich geworden sein: im Kontext einer zunehmenden kulturellen Globalisierung lassen sich „globalisierte Medienszenen“ von Jugendlichen rekonstruieren. Die empirische Forschung wird versuchen müssen, die Strukturen dieser neuen, glokal und transnational durchdrungenen Lebenszusammenhänge besser als bisher sichtbar zu machen. Der Erfolg einer solchen Erforschung wäre dann erzielt, wenn die abstrakte Kategorie der Globalisierung etwa am Beispiel der globalisierten Medienszenen von deutsch-türkischen Jugendlichen im Netz konkret gemacht werden könnte.

\section{Literatur}

Androutsopoulos, J.: Einleitung. In: Ders. (Hrsg.): HipHop: Globale Kultur - lokale Praktiken. Bielefeld 2003a, S. 9-23.

Androutsopoulos, J.: Musikszenen im Netz: Felder, Nutzer, Codes. In: Merkens, H./Zinnecker, J. (Hrsg.): Jahrbuch Jugendforschung 2003. Bielefeld 2003b, S. 57-82.

Androutsopoulos, J./Hinnenkamp, V.: Code-Switching in der bilingualen Chat-Kommunikation: ein explorativer Blick auf \#hellas und \#turks. In: Beißwenger, M. (Hrsg.): Chat-Kommunikation: Sprache, Interaktion, Sozialität \& Identität in synchroner computervermittelter Kommunikation. Stuttgart 2001.

Appadurai, A.: Globale ethnische Räume. In: Beck, U. (Hrsg.): Perspektiven der Weltgesellschaft. Frankfurt a.M. 1998, S. 11-40. 
Asbrand, B.: Globales Lernen und das Scheitern der großen Theorie. Warum wir heute neue Konzepte brauchen. In: ZEP - Zeitschrift für internationale Bildungsforschung und Entwicklungspädagogik, 25. Jg., 2002, H. 3, S. 13-19.

Azrak, A.: Links zum Leben und Überleben. In: medien concret 2002, S. 10-11.

Baacke, D.: Internet, Multimedia und neue Entwicklungsaufgaben. In: GMK(Hrsg.): Rundbrief Themen: Netzwärts - Multimedia und Internet. Bielefeld 1997, S. 3-10.

Baacke, D.: Medienkulturen - Jugendkulturen. In: Radde, M./Sander, U./Vollbrecht, R. (Hrsg.): Jugendzeit - Medienzeit. Weinheim/München 1998, S. 15-42.

Baacke, D.: Die 13-18jährigen. Weinheim und Basel 2000.

Baacke, D./Ferchhoff, W./Vollbrecht, R.: Kinder und Jugendliche in medialen Welten und Netzen. In: J. FritzfW. Fehr (Hrsg.): Handbuch Medien: Computerspiele. Bonn 1999, S. 31-57.

Beck, U.: Was ist Globalisierung? Frankfurt a.M. 1997.

Beck, U.: Macht und Gegenmacht im globalen Zeitalter. Frankfurt a.M. 2002.

Beck, U./Giddens, A./Lash, S.: Reflexive Modernisierung. Eine Kontroverse. Frankfurt a.M. 1996. Bennett, A.: HipHop am Main: Die Lokalisierung von Rap-Musik und HipHop-Kultur. In: Androutsopoulos, J. (Hrsg.): HipHop: Globale Kultur - lokale Praktiken. Bielefeld 2003, S. 26-42.

Bromley, R.: Das Aushandeln von diasporischen Identitäten. In: Hepp, A./Löffelholz, M. (Hrsg.): Grundlagentexte zur transkulturellen Kommunikation. Konstanz 2002, S. 795-818.

Burnett, R./Marshall, P.D.: Web Theory. An introduction. London/New York 2003.

Canclini, N.G.: Hybrid Cultures. New York 1995.

Castells, M.: Das Informationszeitalter. Band I: Die Netzwerkgesellschaft. Opladen 2001.

Cohen, R.: Diasporas and the nation-state: from victims to challlengers. In: International Affairs 72, 1996, S. 507-520.

Cunningham, St./Nguyen, T.: Floating Lives: the Media of the Vietnamese Diaspora. Queensland 1998.

Cunningham, St./Nguyen, T.: Popular Media and the Vietnamese Diaspora. In: Javnost/The Public 6 (1999) 1, S. 71-92.

Dette, C.: Der türkische Cybermuslim in Deutschland - türkisch-deutscher Islam im Internet. In: Becker, J./Behnisch, R. (Hrsg.): Zwischen kultureller Zersplitterung und virtueller Identität. Türkische Medienkultur in Deutschland III. Rehburg-Loccum 2003. S. 135-150.

Deutsche Shell (Hrsg.): Jugend 2002. Frankfurt a.M. 2002.

Featherstone, M.: Global Culture: An Introduction. In: Ders. (Hrsg.): Global Culture. London u.a. 1990, S. 1-14.

Giddens, A.: The Consequences of Modernity. Cambridge 1990.

Giddens, A.: Modernity and Self-identity. Cambridge 1991.

Gillespie, M.: Television, Ethnicity and Cultural Change. London 1995.

Hall, St.: Cultural identity and diaspora. In: Rutherford, J. (Hrsg.): Identity: Community, Culture, Difference. London 1990.

Hannerz, U.: Scenarios for Peripheral Cultures. In: King, A.D. (Hrsg.): Culture, Globalization and the World System. London 1991.

Hepp, A./Löffelholz, M. (Hrsg.): Grundlagentexte zur transkulturellen Kommunikation. Konstanz 2002.

Hornstein, W: Erziehung und Bildung im Zeitalter der Globalisierung. Themen und Fragestellungen erziehungswissenschaftlicher Reflexion. In: Zeitschrift für Pädagogik, 47. Jg., 2001, H. 4, S. 517537.

Huntington, S.P.: The clash of civilizations? In: Foreign Affairs, 1993, vol. 72, S. 23-49. Interview mit Tamer Kulmac „Wir haben ein starkes Wachstumspotential.“ In: medien concret 2002, S. 11.

Kaya, A.: „Scribo Ergo Sum“: Islamic Force und Berlin-Türken. In: Androutsopoulos, J. (Hrsg.): HipHop: Globale Kultur - lokale Praktiken. Bielefeld 2003, S. 246-272.

Kellner, D.: Die erste Cybergeneration. In: SpoKK (Hrsg.): Kursbuch Jugendkultur. Mannheim 1997, S. 310-316.

Lewis, M.W./Wigen, K.E.: The Myth of Continents. Berkely u.a. 1997.

McLuhan, M.: Understanding Media: the Extension of Man. London 1964.

Mandaville, P: Transnational Muslim Politics. London/New York 2001.

Marotzki, W: Online-Ethnographie - Wege und Ergebnisse zur Forschung im Kulturraum Internet. In: Bachmair, B./Diepold, P./de Witt, C. (Hrsg.): Jahrbuch Medienpädagogik 3. Opladen 2003, S. 149165. http://www.medienpaed.com/article/view/524. doi:10.21240/mpaed/retro/2017.07.09.X. 
Mecheril, P./Teo, T. (Hrsg.): Andere Deutsche. Berlin 1994.

Merkens, H./Zinnecker, J.: Jahrbuch Jugendforschung. Opladen 2001.

Mikos, L.: „Interpolation and sampling“: Kulturelles Gedächtnis und Intertextualität im HipHop. In: Androutsopoulos, J. (Hrsg.): HipHop: Globale Kultur - lokale Praktiken. Bielefeld 2003, S. 64-84.

Naficy, H.: The Making of Exile Cultures. Minneapolis 1993.

Nederveen Pieterse, J.: Der Melange-Effekt. In: Beck, U. (Hrsg.): Perspektiven der Weltgesellschaft. Frankfurt a.M. 1998, S. 87-124.

Nelson, B.: Civilizational complexes an intercivilizational encounters. In: Sociological Analysis. 1973, vol. 34, S. 79-105.

Nestvogel, R.: Sozialisation unter Bedingungen von Globalisierung. In: Scheunpflug, A./Hirsch, K (Hrsg.): Globalisierung als Herausforderung für die Pädagogik. Frankfurt a.M. 2000, S. 169- 194.

Özdemir, C.: Viel Bedarf, wenig Angebot. In: Zeitschrift für KulturAustausch 3/1999, S. 15 Portes, AJGuarnizo, L.E./Landolt, $P$ : The study of transnationalism: pitfalls and promise of an

emergent research field. In: Ethnic and Racial Studies 22, 1999, S. 217-237.

Pries, L.: Transnationale Soziale Räume. In: Beck, U. (Hrsg.): Perspektiven der Weltgesellschaft. Frankfurt a.M. 1998, S. 55-86.

Pries, L.: Migration und Integration in Zeiten der Transnationalisierung oder: Warum braucht Deutschland eine ,Kulturrevolution? In: Zeitschrift für Migration und soziale Arbeit, 2001, Heft 1, S. 14-19.

Pries, L.: Transnationalisierung der sozialen Welt? In: Berliner Journal für Soziologie, 2002, Heft 2, S. 263-272.

Rheingold, H.: The Virtual Community. Reading, Mass. 1993.

Robertson, R.: Globalization. London 1992.

Robertson, R.: Glokalisierung: Homogenität und Heterogenität in Raum und Zeit. In: Beck, U (Hrsg.): Perspektiven der Weltgesellschaft. Frankfurt a.M. 1998, S. 192-220.

Roth, R: Globalisierungsprozesse und Jugendkulturen. In: Aus Politik und Zeitgeschichte B5/ 2002, S. 20-27

Sassen, S.: The Global City: New York, London, Tokyo. Princeton/NJ 1991.

Scherer, H./Wirth, W: Ich chatte - wer bin ich? Identität und Selbstdarstellung in virtuellen Kommunikationssituationen. In: Medien\&Kommunikationswissenschaft 50, Jg. 2002/3. S. 337- 358.

Scheunpflug, A.: Stichwort Globalisierung und Erziehungswissenschaft. In: Zeitschrift für Erziehungswissenschaft, 6. Jg., H. 2/2003, S. 159-172.

Schiller, H.: Mass Communications an American Empire. New York 1970.

Schulte, J.: Die Internet-Nutzung von Deutsch-Türken. In: Becker, J./Behnisch, R. (Hrsg.): Zwischen kultureller Zersplitterung und virtueller Identität. Türkische Medienkultur in Deutschland III. Rehburg-Loccum 2003. S. 115-123.

Schwann, K: Breakdance, Beats und Bodrum. Türkische Jugendkultur. Wien 2002.

Senay, U: Virtuelle Welten für Migranten im World Wide Web. In: Becker, J./Behnisch, R. (Hrsg.): Zwischen kultureller Zersplitterung und virtueller Identität. Türkische Medienkultur in Deutschland III. Rehburg-Loccum 2003. S. 125-134.

Silverstone, R: Minderheiten, Medien und die globale Allmende. In: Hepp, A./Löfflelholz, M. (Hrsg.): Grundlagentexte zur transkulturellen Kommunikation. Konstanz 2002, S. 725-749.

Slouka, M.: War of the Worlds: Cyberspace and the High-Tech Assault on Reality. New York 1995.

Smith, M.P.: Transnational Urbanism. Malden/MA 2001.

Sreberny, A.: Media and Diasporic Consciousness: An Exploration among Iranians in London. In: Cottle, S. (Hrsg.): Ethnic Minorities and the Media. Buckingham u.a., 2000, S. 179-196.

Stegbauer, Chr.: Grenzen virtueller Gemeinschaft. Wiesbaden. 2001.

Stegers, E: Portale für Migranten. In: onlinejournalismus.de. [http://www.ojour.de/druckversion/webwatch/migranten.html; 16.09.2003]

Tapscott, D.: Net Kids. Wiesbaden 1998.

Tomlinson, J.; Internationalismus, Globalisierung und kultureller Imperialismus. In: Hepp, A./Löffelholz, M. (Hrsg.): Grundlagentexte zur transkulturellen Kommunikation. Konstanz 2002, S. 140163.

Turkle, S.: Leben im Netz. Reinbek 1998.

Vogelgesang, W: Jugendkulturelle Identitätsinszenierung und Szenengenerierung im Internet. In: Berliner Journal für Soziologie, Heft 1, 1999, S. 65-84. 
Vogelgesang, W: Das Internet als jugendkultureller Erlebnisraum. In: Marotzki, W./Meister, D.MJ Sander, U. (Hrsg.): Zum Bildungswert des Internet. Opladen 2000, S. 363-385.

Volkmer, I.: News in the Global Sphere. Luton 1999.

Volkmer, I: The Global Network Society and the Global Public Sphere. In: development, Vol. 46/ 1 (2003). pp. 9-16.

Vollbrecht, R: Einführung in die Medienpädagogik. Weinheim und Basel 2001.

Vollbrecht, R: Jugendmedien. Tübingen 2002.

Wallerstein, I.: The Politics of the World Economy: the States, the Movements and the Civilizations. Cambridge 1987.

Weiß, H.-J./Trebbe, J.: Mediennutzung und Integration der türkischen Bevölkerung in Deutschland. Potsdam 2001.

Wellman, B./Gulia, M.: Virtual Communities as Communities. Net surfers don’t ride alone. In: Smith, M.A./Kollock, P. (Hrsg.): Communities in Cyberspace. Routledge 1999, S. 167-194.

Welsch, W: Transkulturalität. Lebensformen nach der Auflösung der Kulturen. In: Information Philosophie 2/1992, S. 5-20. 